\title{
Cataracte et rayonnements ionisants
}

\author{
S. WASSILIEFF ${ }^{1}$
}

(Manuscrit reçu le 25 juin 2009, accepté le 18 septembre 2009)

RÉSUMÉ La cataracte radio-induite était considérée jusqu’à nos jours comme une pathologie assez peu fréquente, nécessitant de fortes doses de rayonnement (dépassant un seuil élevé, de l'ordre de 2 Grays au cristallin) et se réduisant principalement aux cataractes radiques des patients de radiothérapie. Plusieurs études récentes portant sur des populations aussi diverses que les astronautes, les survivants d'Hiroshima-Nagasaki, les patients ayant subi un scanner céphalique, les « liquidateurs » de Tchernobyl ainsi que quelques expérimentations animales nous amènent à reconsidérer la question : le seuil d'apparition aussi bien des opacités détectables que des cataractes symptomatiques paraît nettement plus bas qu'actuellement estimé. L'existence même d'un seuil n'est plus tout à fait une certitude dans la mesure où la pathogénie de la cataracte radio-induite serait moins de type déterministe (dommage tissulaire direct tuant ou endommageant gravement une population cellulaire donnée) comme on le pensait, mais d'avantage de type stochastique (altération du génome des cellules cibles, perturbation de la division cellulaire, trouble de la différenciation cellulaire des cellules filles). De manière plus pratique, ces observations sont de nature à nous faire reconsidérer la protection des populations spécifiquement exposées : patients et travailleurs principalement. S'agissant des travailleurs, et si ces nouvelles données étaient confirmées, la limite actuelle de dose équivalente au cristallin du code du travail de $150 \mathrm{mSv}$ sur 12 mois consécutifs pourrait, à terme, être revue à la baisse.

ABSTRACT Cataract and ionizing radiation.

The radiation-induced cataract has been up to now considered as a quite rare pathology, needing high-dose radiations (beyond a dose threshold roughly estimated at 2 Grays to the lens) consisting mainly in head tumour radiotherapy complications. Several new studies on different exposed populations such as astronauts, japanese atomic bomb survivors, people undergoing $X$-ray examinations, Chernobyl accident "liquidators" as well as data from animal experiments, suggest that dose threshold for detectable opacities as well as for clinical posterior subcapsular cataract occurring, might be far lower than those previously assumed. Even the existence of a dose threshold is no longer an absolute certitude insofar as radiation-induced cataract pathogeny might consist not really in a deterministic effect (direct tissue harmful effect, killing or seriously injuring a critical population of cells) as believed until now, but rather in a stochastic effect (genomic damage in target-cells, altered cell division, abnormal lens fiber cell differentiation). More practically, these new data may lead us to reconsider radioprotection of specifically exposed populations: mainly patients and workers. Regarding workers, labour legislation (lens equivalent dose limit of $150 \mathrm{mSv}$ during 12 consecutive months) might be, in the medium term, reassessed downwards.

Keywords: Cataract / ionizing radiations / threshold dose / radioprotection

1 École des applications militaires de l'énergie atomique, CC 19, 50115 Cherbourg Octeville Cedex, France. 


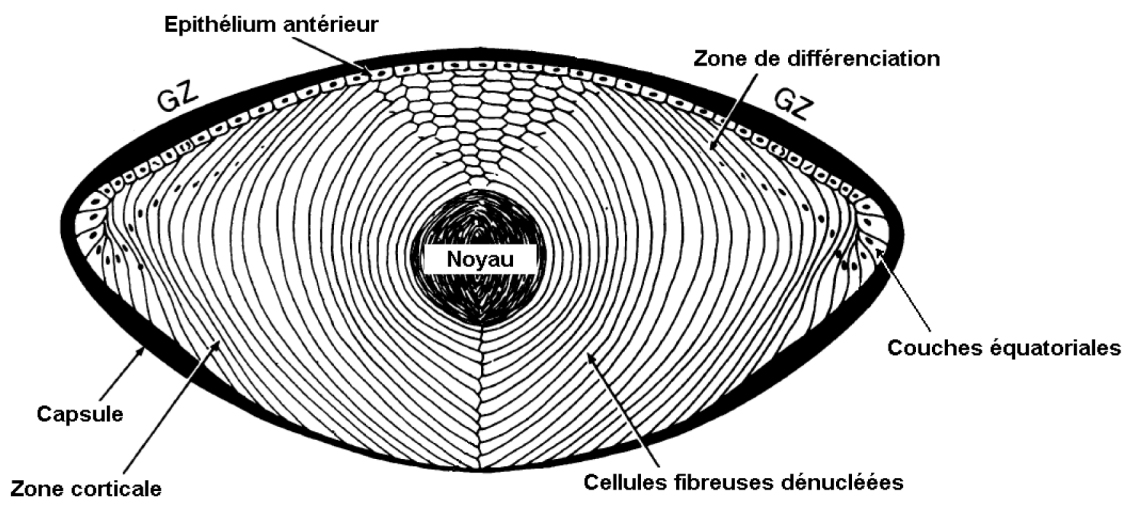

Figure 1 - Coupe sagittale du cristallin (d'après Merriam et Worgul, 1983), GZ = Zone germinative A diagram of a sagittal section of a human lens illustrating the various cellular relationships in that tissue, $\mathrm{GZ}=$ germinative zone.

\section{Introduction}

La cataracte se définit comme une opacification du cristallin entraînant une baisse d'acuité visuelle. Parmi les étiologies de la cataracte, l'action des rayonnements ionisants, connue depuis un siècle, constitue une entité originale. Un certain nombre d'études récemment publiées semblant être à même de remettre en cause quelques notions établies concernant ces cataractes radio-induites, cet article se propose de faire le point de la question.

Pour cela, après avoir exposé quelques brèves notions d'anatomie et de physiologie du cristallin, nous présenterons rapidement les différents types de cataracte et leur traitement, puis nous tâcherons de préciser le mécanisme physiopathologique de la cataracte radio-induite ainsi que ses principales spécificités. Nous passerons ensuite en revue plusieurs données récentes de la littérature et tenterons d'en tirer quelques enseignements concernant la radioprotection.

\section{Rappels}

\subsection{Le cristallin : notions d'anatomie et de physiologie (Fig. 1)}

Le cristallin est une lentille biconvexe transparente et avasculaire suspendue au corps ciliaire par les fibres de la zonule ; ses rapports se font avec l'iris et l'humeur aqueuse en avant et le corps vitré en arrière.

Il joue un rôle optique important car c'est un dioptre de 21 dioptries ; de plus, de façon optimale chez le sujet jeune, sa plasticité est telle que l'action des muscles ciliaires peut modifier ses rayons de courbure permettant ainsi le phénomène d'accommodation. 
Le cristallin est donc un organe avasculaire dont tous les échanges s'effectuent par diffusion à travers sa capsule ou cristalloïde, membrane conjonctive fine et transparente dans laquelle il est totalement ensaché. D'un diamètre frontal de $9 \mathrm{~mm}$ et de $4 \mathrm{~mm}$ d'épaisseur, cette lentille présente une structure en bulbe d'oignon constituée de couches concentriques s'enroulant les unes autour des autres et ce tout au long de la vie.

Ces différentes couches résultent de la prolifération de l'épithélium antérieur : couche unicellulaire directement au contact de la capsule antérieure dont les cellules se multiplient puis migrent, s'étirent au niveau de l'équateur cristallinien, et perdent progressivement leur noyau (différenciation) pour former des fibres (ou cellules fibreuses). Cette zone germinative, située à l'avant du cristallin, est active tout au long de la vie faisant "grossir » le cristallin ; les plus jeunes des fibres ainsi créées refoulant vers le centre les fibres plus anciennes. L'examen à la lampe à fente (ou biomicroscopie) permet une excellente analyse des différents noyaux constituant le cristallin, noyau embryonnaire en son centre entouré par le noyau fœtal puis adulte et la zone corticale répondant immédiatement à la cristalloïde.

\subsection{Les cataractes : principaux types et traitement (Hamard et Hamard, 1993)}

(1) La cataracte sénile : c'est la plus fréquente, $50 \%$ de la population de race blanche de plus de 60 ans présente un certain degré d'opacification du cristallin. Elle est souvent nucléaire, par sclérose des protéines du noyau, volontiers bilatérale et asymétrique. Elle peut être corticale, occupant habituellement la partie équatoriale du cortex cristallinien (opacités cunéiformes « en cavaliers », épargnant longtemps l'axe optique).

(2) La cataracte traumatique : touchant classiquement le sujet jeune, le plus souvent unilatérale. Par contusion, elle est volontiers corticale postérieure voire sous-capsulaire postérieure. Par traumatisme perforant, elle sera unilatérale, s'accompagnera, le plus souvent, d'autres lésions visibles du globe. Sa découverte fortuite impose la recherche d'un corps étranger intraoculaire méconnu.

(3) Les autres étiologies sont principalement: le diabète, la corticothérapie générale de longue durée ( $>1$ an), les cataractes congénitales.

(4) Le traitement est uniquement chirurgical : lorsque la gêne fonctionnelle le nécessite, extraction du cristallin sous anesthésie locale et mise en place d'un implant intraoculaire. 


\subsection{Physiopathologie de la cataracte radio-induite (Merriam et Worgul, 1983)}

Les cellules épithéliales de la zone germinative (région sous capsulaire antérieure paramédiane du cristallin) vont constituer les cibles spécifiques des rayonnements ionisants (Fig. 1). L'irradiation va d'abord être suivie d'une inhibition, de durée variable, de l'activité mitotique de ces cellules. Quand la mitose reprend, et au cours des semaines suivant l'irradiation, les cellules filles des cellules irradiées vont migrer vers les «couches équatoriales » du cristallin où un premier aspect pathologique est constitué par une désorganisation de l'agencement normalement très régulier de ces cellules à cet endroit, premier signe d'une altération de la différenciation cellulaire. Par la suite, la migration se poursuivant, des cellules fibreuses dysmorphiques encore nucléées, vont commencer à s'accumuler dans la région sous-capsulaire postérieure. Ces cellules, qui vont prendre une forme arrondie avec un aspect «en vessie », présentent souvent un noyau pycnotique (annonçant leur mort prochaine) et se nomment cellules de Wedl. Ces cellules de Wedl vont ensuite se rompre, déverser leur contenu éosinophile et répandre des débris cellulaires parmi d'autres cellules apparemment intactes. Le cristallin étant avasculaire et totalement enclos dans sa capsule, ces débris cellulaires ne peuvent être évacués.

Pendant que se produisent ces phénomènes dans le cortex périphérique postérieur, les fibres plus internes semblent garder une morphologie normale. Des cellules dysmorphiques peuvent aussi commencer à apparaître dans le cortex antérieur, finissant éventuellement par occuper le pourtour du cortex.

Ces modifications cyto-architecturales sont à la base de l'altération de la transparence du cristallin.

\section{Aspects historique, clinique, pathogénique et réglementaire de la cataracte radio-induite}

Dès la fin du XIX ${ }^{\mathrm{e}}$ siècle, Chalupecky suggérait que les rayons $\mathrm{X}$, récemment découverts, pouvaient provoquer une cataracte (Chalupecky, 1897). Trente ans plus tard, Rohrschneider en publie la première description clinique : opacités de la région sous-capsulaire postérieure constituant une sorte de plaque comportant des granulations et des vacuoles, de teinte parfois jaunâtre et d' aspect « en cuivre battu » (Rohrschneider, 1930) (Fig. 2). Par la suite le développement de la radiothérapie va voir apparaître les cataractes radiques, pathologie iatrogène des traitements des tumeurs de la face (exemple : paupières) ou de l'orbite (métastases choroïdiennes) notamment. 


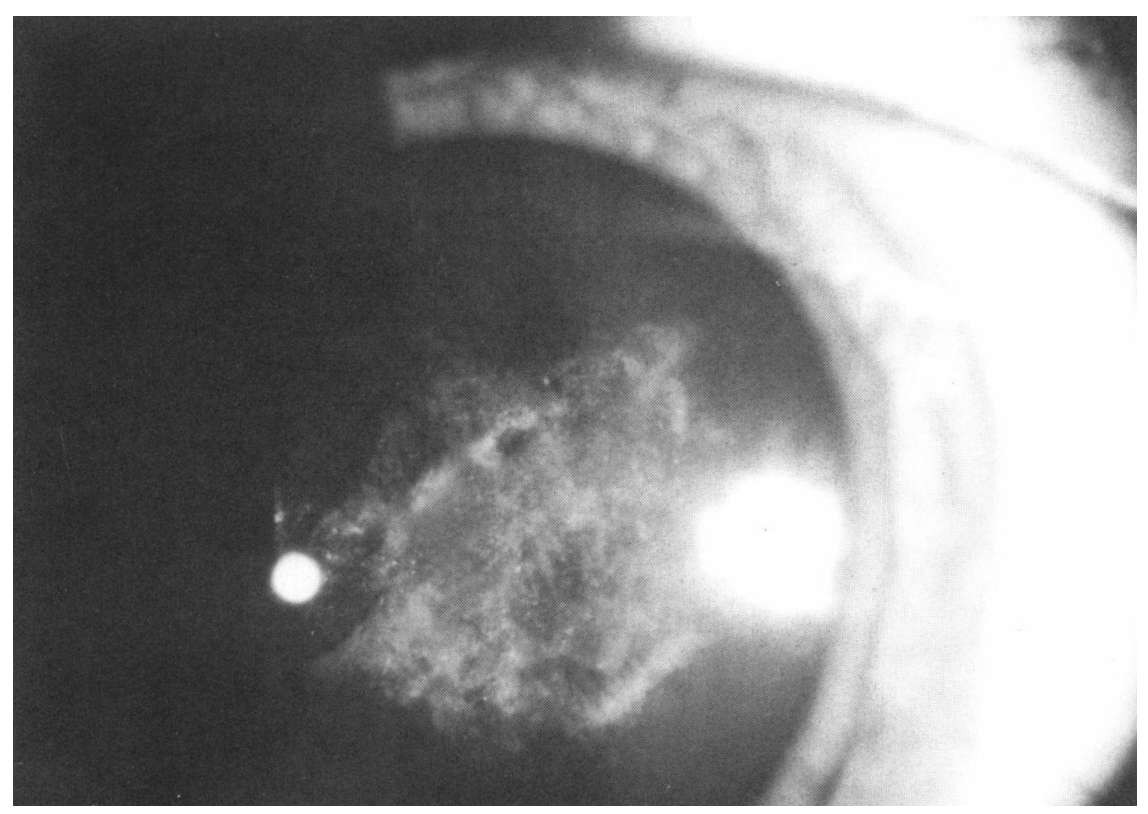

Figure 2 - Aspect de cataracte radio-induite (sous-capsulaire postérieure) à la lampe à fente (d'après Merriam et Worgul, 1983).

A slit-lamp photomicrograph of a typical human radiation cataract. The opacity is located in the subcapsular region of the lens.

\subsection{Dose minimale susceptible de provoquer une cataracte radio-induite}

En 1950, Merriam proposait une fourchette entre 5 et 15 Grays (Merriam et Worgul, 1983). En 1957, Merriam et Focht, se servant d'un mannequin anthropomorphique (crâne de squelette humain recouvert de cire et possédant un cristallin artificiel muni d'un dispositif dosimétrique dans l'orbite), pouvaient réaliser une dosimétrie comparative relativement précise des doses délivrées au cristallin des porteurs de diverses tumeurs de la face : ils proposaient un seuil de 2 Gy en dose unique et de 4 Gy en dose fractionnée et/ou étalée dans le temps (Merriam et Focht, 1957).

\subsection{Délai d'apparition}

Il est inversement proportionnel à la dose et allongé par le fractionnement des doses. Il peut varier entre 6 mois et 35 ans; la moyenne serait environ de 2 à 3 ans (Merriam et Worgul, 1983). 


\subsection{Influence de l'âge}

Dès 1907, des expériences animales suggéraient que les jeunes cristallins étaient plus radiosensibles (Tribondeau et Lafargue, 1907). Toutefois des expériences postérieures sur des rats albinos affinaient cette donnée en montrant que cette radiosensibilité spécifique augmentait avec la dose (Merriam et Szechter, 1973).

\subsection{Influence de la nature des rayonnements}

S'agissant de la cataractogénicité, des expériences sur des cristallins de rats ne retrouvent pas de différence entre l'action des $\gamma$ du cobalt-60 et celle des rayons $\mathrm{X}$ d'énergie (maximale) $200 \mathrm{keV}$ (Focht et al., 1968).

Par contre, les expériences sur cristallins de souris montrent que les neutrons semblent, à dose absorbée égale, 4 à 10 fois plus cataractogènes que les rayons $\mathrm{X}$; cette efficacité biologique relative (EBR) spécifique diminuant quand on augmente la dose (Merriam et al., 1965).

\subsection{Aspects réglementaires}

La Commission internationale de protection radiologique (CIPR) considère la cataracte radio-induite comme un effet déterministe des rayonnements ionisants c'est-à-dire comme un dommage tissulaire direct, de gravité proportionnelle à la dose reçue et n'apparaissant qu'à partir d'un certain taux de cellules tuées ou gravement altérées fonctionnellement (ICRP, 1991).

La dose nécessaire pour atteindre ce nombre minimal de cellules tuées ou gravement endommagées au sein du tissu constituant le seuil de l'effet en question. L'ordre de grandeur du seuil de la cataracte radio-induite est estimé à ce jour à environ 2 Grays. En réalité ce seuil varie selon plusieurs paramètres qui sont principalement le type de rayonnement, le débit de dose, la prise en compte de la simple constatation d'opacités visibles à la lampe à fente ou la prise en compte des altérations de la fonction visuelle (cataracte « clinique » ou symptomatique). Plus précisément, la CIPR dans sa publication 103 donne comme seuils (ICRP, 2007) :

- pour les opacités détectables :

$\circ 0,5$ à 2 Gy en exposition brève unique,

○ 5 Gy en dose cumulée pour des expositions très fractionnées ou étalées ;

- pour les altérations de la fonction :

$\circ 5$ Gy pour les expositions uniques,

$\circ>8$ Gy pour les expositions cumulées ;

- du point de vue de la réglementation en radioprotection, le code du travail stipule que : «pour le cristallin, l'exposition reçue au cours de douze mois consécutifs ne peut dépasser $150 \mathrm{mSv} »$ (Code du travail, 2008). 
Un travailleur est considéré comme exposé au risque dans la mesure où il est susceptible de recevoir une dose équivalente au cristallin supérieure à $15 \mathrm{mSv}$ par an (dose « public »). C'est le cas notamment de certains personnels hospitaliers, en radiologie interventionnelle, tout particulièrement, où une dosimétrie cristallinienne peut être réalisée par port sur le front d'une pastille de fluorure de lithium (dosimètre thermoluminescent).

Les doses reçues au cristallin (sans protection) par le travailleur (médecin radiologue vasculaire et gastro-entérologue, entre autres) variant, selon les études et les pratiques, entre 0,05 et 3,2 mSv par examen (Aubert et al., 1997). Le port d'une protection spécifique (lunettes à verres au plomb) est conseillé, malgré son caractère contraignant (poids), pour les examens les plus irradiants surtout s'ils sont fréquents.

Dans les armées, l'instruction de référence prévoit, lors de la visite médicale d'aptitude initiale des personnels affectés à des travaux sous rayonnements ionisants «un examen ophtalmologique de référence pour le personnel de catégorie A devant être soumis de façon prolongée à une exposition externe. Cet examen comporte l'examen des milieux transparents en vue de mettre en évidence d'éventuelles opacités cristalliniennes » (Instruction $n^{\circ}$ 4916, 2009). Cet examen initial sera suivi, dans les cas où il existerait une exposition particulière, par d'autres examens ophtalmologiques réguliers prescrits par le médecin chargé de la surveillance médicoradiobiologique.

\section{Les données récentes de la littérature}

\subsection{Les survivants des bombardements d'Hiroshima et de Nagasaki}

- Une étude cas-témoins de 2004 portant sur des personnes exposées alors qu'elles avaient moins de 13 ans et examinées entre 2000 et 2002 montre, pour une dose estimée au cristallin de $1 \mathrm{~Sv}$, une augmentation de $12 \%$ du taux de cataracte nucléaire (Odds Ratio $(\mathrm{OR})=1,12)$, de $29 \%$ pour les cataractes corticales et de $41 \%$ pour les cataractes sous capsulaires postérieures (CSCP) (Minamoto et al., 2004).

- En 2006, une autre étude cas-témoins japonaise retrouve un taux très proche $(\mathrm{OR} / \mathrm{Sv}=1,44$ avec $1,19<$ intervalle de confiance (IC) à $95 \%<1,73)$ pour les CSCP. La même étude s'intéresse au seuil d'apparition qu'elle estime à $700 \mathrm{mGy}$ pour les $\mathrm{CSCP}$, l'intervalle de confiance à $90 \%$ incluant le 0 ce qui rendrait possible l'hypothèse d'une absence de seuil (Nakashima et al., 2006). 


\subsection{Les cosmonautes}

- Une étude de la NASA portant sur 295 astronautes répartis en 2 groupes a établi une augmentation significative de l'incidence des cataractes d'apparition précoce dans le groupe ayant reçu une dose estimée au cristallin supérieure à $8 \mathrm{mSv}$ (Cucinotta et al., 2001).

- Une étude cas-témoins allemande comparant 21 anciens astronautes et cosmonautes avec 395 témoins d'âge comparable montre une augmentation du taux de cataractes corticales postérieures et sous-capsulaires postérieures chez les premiers (Rastegar et al., 2002).

- Une étude américaine récente a surveillé pendant 5 ans les cristallins de 3 populations différentes : 171 astronautes, 95 personnels volants et 99 personnels des bases. Un taux significativement supérieur de cataractes corticales a été retrouvé chez les astronautes exposés aux radiations spatiales. Des CSCP étant retrouvées également, particulièrement chez les sujets fortement exposés aux rayonnements cosmiques. En revanche aucun excès de cataracte nucléaire n'était constaté chez les astronautes (Chylack et al., 2009).

\subsection{Tchernobyl}

Une étude de cohorte ukraino-américaine (UACOS) est menée depuis 1996 sur les cristallins des « liquidateurs » de Tchernobyl. Il s'agit de personnes ayant travaillé à la décontamination du site durant les mois et les années qui ont suivi l'accident de 1986 (Kleiman, 2007).

Une des récentes publications de cette étude porte sur 8607 liquidateurs pour lesquels une cataracte a été recherchée 12 et 14 ans après l'exposition (Worgul et al., 2007).

La prévalence de cataractes d'allure sénile (cataracte nucléaire) est faible $(3,9 \%)$ ce qui correspond à l'âge moyen de la cohorte (90\% avait moins de 55 ans au premier examen ophtalmologique).

Par contre une cataracte sous-capsulaire postérieure ou corticale, d'allure radio-induite, était retrouvée chez $25 \%$ des sujets examinés.

La même étude établit que, s'agissant de la dose-seuil, elle serait, dans tous les cas, inférieure à $700 \mathrm{mGy}$ et vraisemblablement inférieure à $350 \mathrm{mGy}$ pour les CSCP de «stade 1 » (opacités n'altérant pas la fonction visuelle).

Il est à remarquer que ces chiffres sont très nettement inférieurs à ceux de la publication 60 de la CIPR (1991) qui donne un seuil de dose équivalente cumulée au cristallin pour les expositions étalées dans le temps de 5 Gy pour les opacités détectables. 


\subsection{Cataractes iatrogènes}

Déjà en 1993, une étude américaine portant sur l'examen du cristallin de 4926 sujets établissait une association significative entre la présence d'opacités souscapsulaires postérieures et le fait d'avoir subi dans sa vie un scanner de l'encéphale (Klein et al., 1993). Or, si on regarde l'ordre de grandeur de la dose délivrée au cristallin par un tel examen, on s'aperçoit que celui ci est relativement modéré : Gambini donne 43,4 mGy quand le plan de coupe passe par les orbites (cas le plus irradiant) (Gambini et Granier, 2007).

En 1999, une étude cas-témoins suédoise portait sur 16500 personnes traitées avant l'âge de 18 mois entre 1920 et 1959 par radiothérapie pour des angiomes cutanés: pour une dose de 1 Gy au cristallin, l'augmentation du risque d'apparition d'une opacité sous-capsulaire postérieure est estimée à $50 \%$ (OR = 1,$5 ; 1,10<\mathrm{IC} 95<2,05$ ) et à $35 \%$ pour des opacités corticales (Hall et al., 1999).

Une récente étude de cohorte américaine a suivi 35705 électro manipulateurs en radiologie médicale, âgés de 24 à 44 ans et aux cristallins sains au début de l'étude, de 1983 à 2004 (Chodick et al., 2008).

Deux types d'exposition ont été simultanément étudiés :

- une exposition médicale : le fait d'avoir subi eux-mêmes 3 ou plus examens radiologiques de la face et/ou du cou était corrélé avec un Risque Relatif (R.R.) de 1,25 d'apparition d'une cataracte $(1,06<$ IC95 < 1,47) soit une augmentation de $25 \%$;

- une exposition professionnelle : 2 groupes ont été définis ; l'un ayant reçu en moyenne $60 \mathrm{mGy}$ au cristallin, l'autre $5 \mathrm{mGy}$.

Un R.R. de $1,18(0,99<$ IC95 < 1,4) soit un excès de risque relatif de $18 \%$ d'apparition d'une cataracte était retrouvé dans le groupe le plus exposé.

\subsection{Expérimentations animales}

Outre les expériences citées plus haut, citons les études réalisées à l'accélérateur d'ions lourds de Californie (BEVALAC) : des rats de 4 semaines ont été irradiés au niveau de la tête par des faisceaux d'ions d'argon-40, particules lourdes de très haute énergie (de l'ordre de 22 Gigaélectronvolts) et de très fort transfert linéique d'énergie (TLE) ; un autre groupe de rats ayant reçu des rayons X (faible TLE). Les ions lourds ont paru de 3,5 à 100 fois plus cataractogènes que les rayons $\mathrm{X}$ (Worgul et al., 1981). Cette observation est à rapprochée du fait que nombre d'astronautes (notamment ceux d'Apollo 11) ont rapporté avoir vu des éclairs et des traits lumineux pendant leur vol. Ces perceptions lumineuses étant probablement dues à des particules lourdes de très haute énergie frappant la rétine. 
Une expérimentation plus récente montre qu'une dose de $100 \mathrm{mGy}$ de rayons X est cataractogène pour la souris (Worgul et al., 2005).

\section{Tableau récapitulatif et discussion}

\begin{tabular}{|c|c|c|c|c|c|}
\hline & $\begin{array}{l}\text { Hiroshima } \\
\text { Nagasaki } \\
(1 \mathrm{~Gy})\end{array}$ & Cosmonautes & Tchernobyl & $\begin{array}{c}\text { Iatrogène } \\
\text { (TDM encéphale) }\end{array}$ & $\begin{array}{c}\text { Iatrogène } \\
\text { (radiothérapie } 1 \\
\text { Gy) }\end{array}$ \\
\hline \multirow{3}{*}{$\begin{array}{l}\text { Type de } \\
\text { cataracte }\end{array}$} & $\pi 12 \% \mathrm{CN}$ & Pas de $\mathrm{CN}$ & $\pi 3,9 \% \mathrm{CN}$ & & \\
\hline & $\pi 29 \% \mathrm{CC}$ & $\pi \mathrm{CC}$ & $(\mathrm{CC}$ & & $\pi 35 \% \mathrm{CC}$ \\
\hline & $\pi 41 \% \mathrm{CSCP}$ & $\pi \mathrm{CSCP}$ & $\mathrm{CSCP}$ & $\pi \mathrm{CSCP}$ & 入 $50 \% \mathrm{CSCP}$ \\
\hline $\begin{array}{l}\text { Seuil estimé } \\
\text { (cataracte) }\end{array}$ & 700 mGy & $>8 \mathrm{mSv}$ & $<700 \mathrm{mGy}$ & & \\
\hline $\begin{array}{l}\text { Seuil estimé } \\
\text { (opacités) }\end{array}$ & & & $<350 \mathrm{mGy}$ & $<43 \mathrm{mGy}$ & \\
\hline
\end{tabular}

$\mathrm{CN}$ : cataracte nucléaire - CC : cataracte corticale - CSCP : cataracte sous-capsulaire postérieure.

Que conclure de ces nouvelles données ?

- S'agissant des seuils d'apparition, il semble bien qu'ils soient nettement inférieurs à ceux retenus jusqu'à présent aussi bien pour les opacités asymptomatiques que pour les cataractes constituées.

- S'agissant du type de cataracte : la cataracte radio-induite est sous capsulaire postérieure ou corticale, pratiquement jamais nucléaire et, logiquement, la plupart du temps, bilatérale.

- S'agissant du caractère déterministe des cataractes radio-induites, celui-ci pourrait même être remis en question dans la mesure où :

○ l'existence même d'un seuil n'est plus tout à fait certaine ;

o l'importance des atteintes génomiques (présence de nombreux micronoyaux) des cellules de l'épithélium antérieur semble prépondérante par rapport aux atteintes cellulaires directes (effet de mort cellulaire) (Worgul et al., 1991).

Ce dommage génotoxique, avec altération de la division cellulaire, semble bien être transmis aux cellules filles qui voient leur différenciation perturbée.

Ceci rapproche ce mode pathogénique de celui des effets stochastiques : en effet la publication 60 de la CIPR (1991) définit l'effet stochastique comme le résultat du « développement d'un clone à partir d'une cellule modifiée ». Cela signifie qu'une cellule dont l'ADN a été endommagé par une radiation et mal réparé va transmettre aux cellules de sa lignée une altération viable et transmissible 
de son génome (mutation) qui pourra à terme se manifester par divers dysfonctionnements cellulaires et tissulaires ; (le plus redouté étant un processus de cancérisation qui ne nous concerne pas ici). Ces effets n'ont théoriquement pas de seuil et l'augmentation de la dose augmente non pas la gravité de l'effet (comme c'est le cas pour les effets déterministes) mais la probabilité de survenue de l'effet. On voit donc que la cataracte radio-induite, par sa pathogénie originale, possède à la fois :

- certaines spécificités des effets déterministes : présence probable (mais non plus certaine) d'un seuil, une certaine proportionnalité de la gravité de l'effet avec la dose ;

- certaines spécificités des effets stochastiques : dommage génomique initial qui ne tue ni ne lèse gravement la cellule cible mais qui va être transmis aux cellules de la lignée.

Tous ces éléments sont de nature à nous faire reconsidérer la stratégie actuelle de la prévention du risque cataracte.

Il semble qu'on ne puisse plus se contenter de respecter des limites de dose équivalente choisies en dessous des seuils d'apparition, comme on le fait légitimement pour les effets déterministes classiques type « brûlure radiologique », d'autant plus que ces seuils semblent avoir été sous-évalués.

L'application du principe d'optimisation de la protection et la surveillance des populations spécifiquement exposées à ce risque retrouvent là toute leur valeur.

\section{Conclusion}

Sous réserve de confirmation par des études ultérieures, les cataractes radioinduites, à la lumière des récentes publications, paraissent devoir être considérées désormais comme une pathologie plus fréquente qu'on ne le pensait. En particulier les seuils d'apparition prévisible méritent probablement d'être réévalués.

En conséquence, la protection et la surveillance des populations exposées à ce risque spécifique (essentiellement patients et travailleurs) devra être, le cas échéant, renforcée.

S'agissant des travailleurs, il n'est pas impossible que la limite réglementaire actuelle de $150 \mathrm{mSv}$ de dose équivalente au cristallin sur 12 mois consécutifs, soit, à terme, revue à la baisse.

Remerciements. Au docteur J.-L. Kovalski pour ses conseils ophtalmologiques. À monsieur Y. Lecorvaisier pour son appui technique. 


\section{RÉFÉRENCES}

Aubert B., Paul D., Sabattier R. (1997) Dosimétrie du personnel en milieu hospitalier, Radioprotection 32, $163-179$.

Chalupecky H. (1897) Ueber die Wirkung der rontgenstrahlen auf das Aufe und die Haut, Z. Prakt. Augenheilk (Leipzig) 21, 234-239.

Chodick G. et al. (2008) Risk of cataract after exposure to low doses of ionizing radiation: A 20-year prospective cohort study among US radiologic Technologists, Am. J. Epidemiol. 168, 620-631.

Chylack L.T. Jr. et al. (2009): NASA Study of cataract in astronauts (NASCA), Report 1: Crosssectional study of the relationship of exposure to space radiation and risk of lens opacity, Rad. Res. 172, 10-20.

Code du travail (2008) Edition du $1^{\text {er }}$ mai 2008 - Article R.4451-13.

Cucinotta F.A., Manuel F.K., Jones J., Iszard G., Murrey J., Djojonegro B., Wear M.V. (2001) Space radiation and cataracts in astronauts, Rad. Res. 156, 460-466.

Focht E.F., Merriam G.R. Jr., Schwartz M.S., Parsons R.W. (1968) The relative biologic effectiveness of cobalt 60 gamma and $200 \mathrm{KV} \mathrm{X-radiation} \mathrm{for} \mathrm{cataract} \mathrm{induction,} \mathrm{Am.} \mathrm{J.} \mathrm{Roentgenol.} \mathrm{Rad.}$ Ther. Nuc. Med. 102, 71-80.

Gambini D.J., Granier R. (2007) Manuel pratique de radioprotection $-3^{e}$ édition, Lavoisier.

Hall P., Granath F., Lundell M., Olsson K., Holm L.E. (1999) Lenticular opacities in individuals exposed to ionizing radiation in infancy, Rad. Res. 152, 190-195.

Hamard P., Hamard H. (1993) Séméiologie et formes cliniques de la cataracte chez l'adulte. Éditions Techniques. Encycl. Méd. Chir. (Paris-France), Ophtalmologie, 21-250-A-30, 6 p.

ICRP Publication 60 (1991) 1990 Recommendations of the International Commission on Radiological Protection - Users' Edition, Ann. ICRP 21(1-3).

ICRP Publication 103 (2007) Recommendations of the ICRP, Ann. ICRP 37(2-4).

Instruction $\mathrm{n}^{\circ}$ 4916/DEF/CAB du 30 mars 2009 relative aux dispositions communes de radioprotection du personnel du ministère la défense ( $\$$ 4.3.1. du guide annexé).

Kleiman N.J. (2007) Radiation cataract, Radiation Protection 145, 81-95.

Klein B.E.K., Klein R., Linton K.L.P., Franke T. (1993) Diagnostic X-ray exposure and lens opacities: The Beaver Eye Dam eye study, Am. J. Publ. Health 83, 588-590.

Merriam G.R. Jr., Focht E.F. (1957) A clinical study of radiation cataracts and the relationship to dose, Am. J. Roentgenol. 77, 759-785.

Merriam, G.R. Jr., Szechter A. (1973) The effect of age on the radiosensitivity of rat lenses, Trans. Am. Ophthal. Soc. 71, 88-110.

Merriam G.R. Jr., Worgul B.V. (1983) Experimental radiation cataract - Its clinical relevance, Bull. N.Y. Acad. Med. 59, 372-392.

Merriam G.R. Jr., Biavati B.J., Bateman J.L., et al. (1965) The dependence of RBE on the energy of fast neutrons. IV Induction of lens opacities in mice, Rad. Res. 25, 123-38.

Minamoto A., Taniguchi H., Yoshitani N., Mukai S., Yokoyama T., Kumagami T., Tsuda Y., Mishima H.K., Amemiya T., Nakashima E., Neriishi K., Hida A., Fujiwara S., Suzuki G., Akahoshi M. (2004) Cataract in atomic bomb survivors, Int. J. Radiat. Biol. 80, 339-345.

Nakashima E., Neriishi K., Minamoto A. (2006) A reanalysis of atomic-bomb cataract data, 20002002: A threshold analysis, Health Phys. 90, 154-160.

Rastegar Z.N., Eckart P., Mertz M. (2002) Radiation cataracts in astronauts and cosmonauts, Graefe. Arch. Clin. Exp. Ophthalmol. 240, 543-547.

Rohrschneider W. (1930) Experimentelle Katarakt Nach Mehrfacher Bestrablung Mit Kleinen Rontgenstrahlendosen, Ophth. Gesellach. 48241-48244. 


\section{CATARACTE ET RAYONNEMENTS IONISANTS}

Tribondeau L., Lafargue P. (1907) Action différente des rayons X sur le cristallin des animaux jeunes et des animaux adultes, C. R. Soc. Biol. 63, 716-717.

Worgul B.V., Merriam G.R. Jr., Medvedovsky C., et al. (1981) The cataractogenic effect of accelerated Argon ions on the rat lens, Rad. Res. 87, 461.

Worgul B.V., David J., Odrich S., Merriam G.R. Jr., Medvedovsky C., Merriam J.C., Trokel S.L., Geard C.R. (1991) Evidence of genotoxic damage in cataractous lenses, Mutagenesis 6, 495499.

Worgul B.V., Smilenov L., Brenner D.J., Vazquez M., Hall E.J. (2005) Mice heterozygous for the ATM gene are more sensitive to both X-ray and heavy ion exposure than are wildtypes, $A d v$. Space. Res. 35, 254-225.

Worgul B.V., Kundiev Y.I., Sergiyenko N.M., Chumak V.V., Vitte P.M., Medvedovsky C., Bakhanova E.V., Junk A.K., Kyrychenko O.Y., Musijachenko N.V., Shylo S.A., Vitte O.P., Xu S., Xue X., Shore R.E. (2007) Cataracts among Chernobyl clean-up workers: implications regarding permissible eye exposures, Rad. Res. 167, 233-243. 